Itinéraires Itinéraires

Littérature, textes, cultures

\title{
Alice Krieg-Planque, La Notion de " formule » en analyse du discours. Cadre théorique et méthodologique
}

Besançon, Presses universitaires de Franche-Comté, 2009

\section{Abdoulaye Diouf}

\section{(2) OpenEdition}

\section{Journals}

Édition électronique

URL : http://journals.openedition.org/itineraires/196

DOI : $10.4000 /$ itineraires. 196

ISSN : 2427-920X

Éditeur

Pléiade

Édition imprimée

Date de publication : 1 juillet 2011

Pagination : 180-186

ISBN : 978-2-296-54673-8

ISSN : 2100-1340

Référence électronique

Abdoulaye Diouf, « Alice Krieg-Planque, La Notion de «formule » en analyse du discours. Cadre théorique et méthodologique », Itinéraires [En ligne], 2011-2 | 2011, mis en ligne le 13 janvier 2014, consulté le 22 septembre 2020. URL : http://journals.openedition.org/itineraires/196 ; DOI : https://doi.org/10.4000/ itineraires. 196 
relations intrinsèques (avec les signifiants, les énonciateurs et les énonciataires) et illustrent bien l'idée selon laquelle le signe fait émerger le corps, le corps fait émerger le signe, et que la signifiance naît de cette réciprocité.

\author{
Marina Krylyschin \\ Université de Paris $5-E D A$ \\ Catherine Ruchon \\ Université Paris 13 - CENEL
}

Alice Krieg-Planque, La Notion de «formule » en analyse du discours. Cadre théorique et méthodologique, Besançon, Presses universitaires de Franche-Comté, 2009, 144 p. ISBN : 978-2-84867-255-7.

Cet ouvrage d'Alice Krieg-Planque fait suite à un premier travail initié en thèse de doctorat en 2000 sur Émergence et emplois de la formule "purification ethnique " dans la presse française (1980-1994) et dont la présentation des résultats et leur analyse en contexte a fait l'objet, trois ans plus tard, d'une publication remaniée aux éditions du CNRS ${ }^{1}$. Elle s'y propose fondamentalement un dessein plus élaboré qui donne une meilleure visibilité au cadre théorique (l' « école française d'analyse du discours ») et méthodologique (détermination des contours de l'objet dans le cadre d'une approche pluridisciplinaire) qui éclaire son analyse de la notion de formule définie comme « un ensemble de formulations qui, du fait de leur emploi à un moment donné et dans un espace public donné, cristallisent des enjeux politiques et sociaux que ces expressions contribuent dans le même temps à construire » (p. 7). Mais au-delà de cette notion de formule, l'ouvrage explore aussi d'autres phénomènes de reprise discursifs comme les « petites phrases » ou les « slogans » qui, tous, permettent de s'apercevoir des rapports interactionnels d'opinion, de domination, etc. qui peuvent exister entre les différents acteurs d'une même sphère socio-politique.

Sur le plan de la structure et de l'architecture, le texte s'organise en cinq chapitres qui interrogent tour à tour et comme suit : des travaux sélectifs sur les usages socio-politiques de mots qui permettent de repérer des formules (chapitre I), le travail heuristique opéré par Jean-Pierre Faye sur la formule État total qui, au-delà de la genèse qu'elle fait du terme de formule même, définit sa modalité de circulation et son caractère constitutivement figé (chapitre II), l'analyse de Marianne Ebel et Pierre Fiala

1. "Purification ethnique ". Une formule et son histoire, Paris, CNRS Éditions, coll. «Communication », 2003, 523 p. 
qui met en place des outils linguistiques (chapitre III) sur la base desquels Alice Krieg-Planque propose quatre propriétés qui spécifient sa conception de la formule (chapitre IV) dont elle fait la synthèse, montre les déplacements (vers la notion d'espace public) et évoque les rapports avec les grands médias (chapitre v).

Dans le chapitre I, « De l'analyse du vocabulaire socio-politique au repérage de formules ", qui procède par rappel de certains travaux de lexicologie socio-politique, la notion de formule est appréhendée comme un phénomène discursif qui vit de l'usage qu'en font les acteurs. Ce sont surtout les analyses d'Alain Rey sur le mot révolution dans "Révolution ». Histoire d'un mot et celles de Marie-France Piguet sur le mot classe dans Classe. Histoire du mot et genèse du concept des Physiocrates aux historiens de la Restauration qui permettent à Alice Krieg-Planque de montrer comment le mot acquiert le statut de formule à la faveur d'usages langagiers marqués par des « heurts de parcours » et des «zones de turbulence ». Ces dernières « secousses sémantiques », pour emprunter le vocabulaire de Marguerite Yourcenar, se donnent surtout à voir à travers l'éparpillement du sens du mot révolution qui peut signifier en même temps " réforme ", « changement constitutionnel », « nouvelle institution », etc., sa productivité lexicologique (avec la naissance de mots dérivés comme «contre-révolution », « révolutionnaire », etc.), les emplois combinés du mot classe dans des syntagmes nominaux ( « la classe productive », « la classe des propriétaires » et « la classe stérile ») ainsi que les opérations métadiscursives qui en résultent et glosant sur le caractère impropre de cette dernière expression. Autant de preuves qui attestent l'existence mouvementée des mots à partir de laquelle Alice Krieg-Planque va tirer des éléments d'analyse qui lui serviront à élaborer les propriétés définitoires de sa conception de la notion de formule : la circulation des termes et la dimension polémique.

Mais c'est dans le chapitre II, « Le travail heuristique de Jean-Pierre Faye : la formule "État total” ", qu' on s'aperçoit plus nettement de l'arrièreplan théorique sur lequel se fonde notre auteur pour mieux affiner les différentes caractéristiques qui donnent forme à sa conception de la formule. Le point de départ est le requestionnement de la notion de « surrécit» mise en route par le philosophe Jean-Pierre Faye pour décrire les conditions de la production et de la circulation des récits et à partir de laquelle Alice KriegPlanque opère un décrochage référentiel où le terme de formule n'est plus utilisé pour désigner uniquement des expressions comme «État total», «État totalitaire », mais renvoie maintenant à des propriétés qu'elle dégage de la formule fayenne totale Staat. La première propriété est la « genèse " du terme depuis la première occurrence de l'adjectif totalitaria dans le discours de Mussolini de 1925 à la notion voisine de « mobilisation totale » théorisée par Ernst Jünger en 1930 pour dire la mobilisation tous azimuts de la société au service de l'État et qui a été reprise par l'idéologue allemand Carl Schmitt pour donner lieu à la formule totale Staat. Genèse qui laisse 
voir la « circulation » (deuxième propriété) géographique du terme (de l'Italie à l'Allemagne en passant par le nord de l'Europe) à laquelle s'ajoute une « circulation » linguistique en termes de productivité lexicologique qui génère des expressions comme totale Revolution, Totale Krise, Dictature totale, etc. Mais quel que soit le changement occasionné par cette mobilité transfrontalière du terme (changement de langue, changement de formation discursive, changement de sens, changement par dérivation ou composition), il y a quand même une certaine stabilité formelle que Krieg-Planque identifie à un terme absent du travail de Jean-Pierre Faye mais proche de la notion de formule, le « figement » (troisième propriété tirée de la formule totale Staat), comme attestation de la soudure de l'expression et, par-delà, du caractère agissant de la langue. Que la formule circule, qu'elle soit attestée, ce sont là des preuves qu'elle est en majorité bien reçue : c'est ce qu'elle appelle « le procès d'acceptabilité » (quatrième propriété tirée de la formule fayenne) comme effet de l'efficacité de la formule destinée à faire accepter volontairement un mot sans besoin de démonstration.

Dans la continuité des analyses de Jean-Pierre Faye, Marianne Ebel et Pierre Fiala proposent des définitions plus précises de la notion de formule à travers les termes Überfremdung ou « emprise et surpopulation étrangères » et « xénophobie », dont Alice-Krieg Planque rend compte dans le chapitre III de son ouvrage. Le contexte historique qui leur sert de prétexte est la Suisse des années 1960-1970, années au cours desquelles le pays a connu trois votations (1970, 1974 et 1977) sur la proposition de limitation de l'immigration. Mais ce qui intéresse fondamentalement notre auteur dans leurs analyses, ce sont les instruments linguistiques, absents des travaux de Faye, qu'ils mettent en place pour construire la notion de formule sur la base de deux séries d'énoncés qui servent à décrire ce que disent les locuteurs : « les énoncés à valeur de re » qui, en se rapportant au contenu référentiel de la formule, attestent son caractère référentiel et « les énoncés à valeur de dicto » qui attestent le caractère polémique de la formule en renvoyant au mode de dire. À partir de ce moment, est formulé, disent-ils en substance, tout signe qui a valeur de référent social, c'est-à-dire qui « signifie quelque chose pour tous à un moment donné ») (p. 55) et qui présente en même temps une dimension « polémique » comme preuve évidente que c'est parce qu'elle est contestée que la formule domine. Au-delà des pistes intéressantes qu'elles ouvrent à la perspective de Krieg-Planque, ces analyses d'Ebel et Fiala opèrent également un déplacement important dans le champ de l'analyse du discours qu'elles pensent en termes d'ouverture et d'hétérogénéité avec les notions de « formule », de « circulation » et de « référent social».

Après ce tour d'horizon d'études qui représentent l'arrière-plan théorique de la notion de formule, Alice Krieg-Planque s'attache, dans l'avant-dernier chapitre de son ouvrage, à ses propres propositions qui circonscrivent le terme en quatre principales propriétés. Pour elle, pour qu'un mot puisse accéder au statut « formulaire », il faut qu'il présente un 
« caractère figé », s'inscrive dans une « dimension discursive », fonctionne comme un « référent social» et comporte un «aspect polémique ». À y regarder de plus près, on se rend compte que l'essentiel de ces propriétés résulte des implications des analyses antérieures qui lui ont servi de source d'inspiration.

À travers le figement (propriété 1), elle veut montrer que la formule s'incarne dans une forme signifiante stable qui facilite son identification. C'est d'ailleurs sur la base de cette exigence de stabilité que les catégories suivantes, parce que procédant selon elle d'un certain relativisme qui leur fait changer de sens selon le contexte socio-culturel, sont écartées de la possibilité d'être des formules : les «préconstruits culturels » de JeanBlaise Grize, les « prédiscours » de Marie-Anne Paveau, les « topoï» de Jean-Claude Anscombre et Oswald Ducrot, les « aphorismes » de MarieAnne Mochet et Iva Cintrat, les « énoncés légiférants » de Blanche-Noëlle et Roland Grunig, et la notion d' « idéologème » de Marc Angenot. Cette forme de la formule, qui peut se présenter en une unité lexicale simple (cas d'un mot) ou complexe (syntagme lexicalisé), en une unité lexicosyntaxique ou en une séquence autonome (slogan), est à prendre en compte, selon Krieg-Planque, sur le double plan morpho-syntaxique (en dehors de toute méthode transformationnelle) et lexical (dans le respect de la singularité du corps de chaque lexème). Mais cette identification à une matérialité linguistique stable ne veut pas pour autant dire que la formule tient du formalisme absolu. L'existence des variantes d'une même formule occasionnée par une productivité lexicologique (comme c'est le cas dans « purification ethnique », " nettoyage ethnique » et " épuration ethnique ») ainsi que celle de formulations concurrentes de la formule sont suffisantes pour dire que le figement ne relève pas d'une approche formaliste.

S'il faut d'abord que la séquence soit linguistiquement descriptible dans une forme stable, il faut par la suite qu'elle soit développée dans des usages langagiers pour qu'elle puisse devenir une formule. Et c'est dans cet usage, qui cache le « caractère discursif » de la formule (propriété 2), que réside la condition fondamentale de son analyse, nous dit Krieg-Planque. C'est pourquoi elle réaffirme que c'est dans l'existence d'un corpus saturé d'énoncés que jaillit le plus souvent la formule, même si l'érection d'une séquence au rang «formulaire » peut coïncider avec ses premières apparitions formelles comme on peut le constater dans les syntagmes néologiques « classe stérile », « purification ethnique » ou dans des emprunts comme glasnost (transparence) et perestroika (reconstruction). Ce qui revient à dire que c'est l'avènement d'un contexte discursif singulier postérieurement à l'existence de la séquence qui est le principal gage du passage de cette dernière au statut de formule. Â titre d'exemple, Krieg-Planque cite l'usage particulièrement abondant des mots « concertation ", " négociation » et « dialogue » dans le contexte discursif des réformes de la Sécurité sociale initiées en 1995 par Alain Juppé, alors que ces mots existaient pourtant tout 
ordinairement dans le vocabulaire socio-politique. Ce qui atteste qu'il s'est opéré une resémantisation (le gouvernement parle de « concertation » et de " dialogue » qui ne sont pas, pour les syndicats qui imposent la « négociation », les vrais termes du débat) qui confirme encore, si besoin en était, la tendance générale de l'existence formelle du mot préalablement à son accès au rang de formule.

En empruntant à Marianne Ebel et Pierre Fiala le caractère de « référent social » de la formule (propriété 3), Alice Krieg-Planque veut montrer son ancrage dans une sphère socio-politique où elle est, en un moment donné, connue de tous. Mais pour que le signe puisse acquérir cette « valeur de re » qui lui permet de renvoyer à un monde commun, nous dit-elle, il doit satisfaire à deux critères majeurs : celui de la «notoriété », qui est quantifiable à travers l'accroissement de sa fréquence certifiée par une forte productivité lexicologique sur un corpus stable (production de dérivés et de mots composés) et celui de son attestation dans des types de discours variés (oraux et écrits comme savants et ordinaires), qui montre sa capacité à transcender différentes formations discursives. De ce fait, la fréquence d'un signe tributaire d'un prétexte mondain et non d'un événement discursif cultivé et entretenu, de même que celle des mots des vocabulaires de spécialité incapables de s'échapper des limites étroites de leur domaine, ne peuvent aucunement, dans sa perspective, être éligibles au statut de formule, même s'ils recouvrent le caractère polémique et de référent commun relativement à leur univers d'appartenance. Bref, «pour que l'on puisse dire que la formule est un signe connu de tous, il faut que l'on puisse en observer la présence dans des types de discours les plus variés possibles. Il faut que les lieux d'émergence de la formule se diversifient. Si la formule est originaire d'une formation discursive, elle doit en sortir » (p. 99).

À ce « caractère de référent social » de la formule, Alice Krieg-Planque associe consubstantiellement, à la suite de Marianne Ebel et Pierre Fiala, un « caractère polémique » (propriété 4) qui est la résultante des séries d'oppositions et d'affrontements qui président à son ancrage dans une sphère socio-politique donnée. Laquelle dimension polémique est la cristallisation des enjeux que cache la formule et qui lui confèrent un caractère " grave », non pas dans le sens dramatique du terme, nous dit-elle, mais dans le sens où elle présente un intérêt commun qui peut même atteindre une proportion nationale. À ce propos, elle donne l'exemple de la valence significative que représente la formule « purification ethnique » dans l'histoire de la Yougoslavie pour montrer encore comment les appréhensions (pour ne pas dire le « caractère polémique ») suscitées par cette désignation présentent la sphère socio-politique et discursive comme un " champ » (au sens bourdieusien du terme) où s'affrontent des mots sous la forme de « guerre de position » et de « guerre de mouvement » selon la métaphore courtinienne qu'elle emploie. À partir de ce moment, la meilleure manière d'étudier une formule consiste à analyser ses opérations métadiscursives 
« opacifiantes » (Jacqueline Authier-Revuz) qui peuvent porter sur plusieurs objets parmi lesquels Alice Krieg-Planque retient : l'injonction de prononciation et du refus d'énoncer (cas du gouvernement Juppé qui évite de prononcer le mot " négociation » préférant ceux de « concertation » et de « dialogue » jugés insuffisants pour les syndicats), la tentative d'appropriation d'une formule monopolisée par une partie adverse, la répudiation d'une formule, l'inadéquation de la formule à la chose qu'elle désigne (l'expression « classe stérile » analysée par Marie-France Piguet), la prédication et le rejet de la prédication (le mot « xénophobe » attribué aux partisans de la lutte contre la « surpopulation étrangère » et qui s'en défendent), le caractère réel ou fantasmé du référent désigné par la formule, etc.

Présenté comme une « synthèse » qui montre le déplacement opéré par Alice Krieg-Planque en retravaillant la notion de formule dans un sens qui trahit le projet de « surrécit » initié par Jean-Pierre Faye, le cinquième et dernier chapitre de l'ouvrage a valeur de conclusion. Il s'articule autour de quatre points.

Le premier point justifie le choix du terme de formule qu'elle a emprunté à Marianne Ebel et Pierre Fiala, qui le tiennent à leur tour du philosophe Jean-Pierre Faye, pour mieux le circonscrire à travers une série de quatre propriétés qui, non seulement le sortent de ce halo d'acceptions diverses et multiples hors du discours scientifique des sciences du langage, mais permettent surtout d'identifier plus clairement son contenu.

Quant au deuxième point, il présente les différentes propriétés qu'elle a énoncées dans son analyse sous la forme d'un continuum qui refuse toutes étanchéités de nature à les mesurer en termes de présence et d'absence. Bien au contraire, la formule est pour elle une catégorie graduelle qui fait que les propriétés sont présentes inégalement, c'est-à-dire à des degrés divers. Le figement, le caractère discursif, le référent social et la dimension polémique étant de ce point de vue tous relatifs en raison respectivement du fait qu'une séquence remplit plus ou moins un certain nombre de tests, que les usages déterminent plus ou moins le statut « formulaire » de la séquence, que certains locuteurs peuvent être réticents à l'usage de la formule qu'ils peuvent éviter à tout prix, et que des séquences peuvent être peu problématiques quand d'autres le sont moyennement ou fortement.

Dans le troisième point, elle opère un déplacement à travers lequel la notion de « référent social » rencontre sur son chemin celle d' « espace public » (mise en place par Jürgen Habermas en 1962) sur la base du principe selon lequel si la formule est connue de tous, c'est parce qu'elle a été relayée par des procédés de publicisation assurés principalement par les grands médias que sont la presse, la radio et la télévision. Pour le dire autrement, la formule a besoin d'être fortement médiatisée par le biais d'un moyen de publicisation pour qu'elle puisse se constituer en « référent social ». Ce qui revient à indexer la responsabilité des médias qu'Alice Krieg-Planque situe, dans le quatrième point du chapitre v qui clôt son 
analyse, plus du côté de la mise en circulation des formules que de leur création. D'ailleurs ils sont concurrencés dans ce rôle d'agent de circulation des formules par ce qu'elle appelle des « pratiques amateures et profanes de communication » que sont les blogs, les forums, les sites participatifs, les pétitions en ligne, les listes de diffusion, etc.

S'il y a, au terme de ce parcours de lecture, une « formule » à retenir fondamentalement de cet ouvrage qui circonscrit scientifiquement et rigoureusement la formule, c'est que les rapports entre les acteurs d'une sphère socio-politique peuvent désormais être lus, en dehors des traditionnels pouvoirs (symboliquement liés au rang ou à l'économie), à travers les pratiques discursives qui règlent les échanges. Ce qui, de ce point de vue, place l'analyse du discours dans une posture assimilable à un carrefour épistémologique.

Abdoulaye Diouf

Université Paris 13 - CENEL

Gilles Boëtsch, Dominique Chevé et Hélène Claudot-Hawad (dir.), Décors des corps, Paris, CNRS Éditions, coll. «Corps », 2010, 397 p. ISBN : 978-2-271-07013-5.

La lecture de Décors des corps laisse un sentiment vivifiant. Cet ouvrage collectif édité par le CNRS sous la direction de Gilles Boëtsch (anthropobiologiste), de Dominique Chevé (anthropologue et philosophe) et de Hélène Claudot-Hawad (anthropologue), ne réunit pas moins de quarante contributions, réparties en quatre grandes sections, et issues pour l'essentiel du colloque Couleurs sur corps, qui rassemblait des chercheurs venus de champs scientifiques très divers ${ }^{1}$. Si le titre laisse attendre un propos plutôt « esthétique » et installe d'emblée une équivoque - linguistique et peut-être conceptuelle - entre le support et l'accessoire, il dérobe stratégiquement le maître-mot de la recherche, précisément pluriel et complexe : la couleur. Le recueil prend en effet pour objet la couleur telle qu'elle s'insère dans la diversité des productions humaines ou, plus précisément, la « mise en couleur » comme pratique de réinvention du corps au carrefour de l'individuel et du communautaire, depuis les systèmes d'organisation sociale les plus anciens jusqu'à l'actualité contemporaine. Cette mise en couleur(s) peut désigner des pratiques éphémères ou irréversibles et s'exerce diversement selon les ingrédients, les matériaux et les techniques utilisés : la peinture, le poudrage, la teinture, le tatouage, les scarifications,

1. Le grand nombre de contributions nous oblige, dans les limites de ce bref parcours, à en relever les résultats substantiels sans mentionner les noms des différents auteurs. 